\title{
Daily Impacts of Missing Teeth in Adult Population in Lahore, Pakistan
}

\author{
Hazik B. Shahzad ${ }^{1}$ \\ Faiza Awais ${ }^{2}$ \\ Noor-Ul-Huda Raza ${ }^{3}$ \\ Hanna A Majeed $^{4}$ \\ Maliha Shahbaz ${ }^{5}$ \\ Farhat Kazmi ${ }^{6}$
}

\author{
BDS, MSc \\ BDS, M.Phil \\ BDS, M.Phil \\ BDS, FCPS \\ BDS, M.Phil \\ BDS, M.Phil, PhD
}

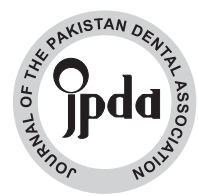

OBJECTIVE: The objective of this study is to evaluate the impact of tooth loss on oral health related quality of life in adults. METHODOLOGY: The survey was conducted during March - June 2019 at Rashid Latif Dental Hospital. A sample size of 373 adults aged 18-80 years was randomly selected from the out-patient department. Number of missing teeth was the main exposure, and it was clinically assessed. OHRQoL was evaluated through "Oral Impacts on Daily performance (OIDP) questionnaire".

RESULTS: From total patients presented in OPD $44 \%$ had one or more missing teeth. Top reported impacts of missing teeth included difficulty eating and relaxing/sleeping problems. Adults with missing teeth showed 1.28 (95\% CI 1.04-4.55) $(\mathrm{P}=0.01)$ times higher odds for reporting higher oral impacts compared to those without missing teeth.

CONCLUSION: Individuals with missing teeth likely had frequent difficulties in their daily lives due to missing teeth including trouble with eating, speaking, and oral infection, which could spread to the rest of your body.

KEYWORDS: OIDP, Tooth-loss, Adults, Missing teeth, Lahore

HOW TO CITE: Shahzad HB, Awais F, Raza NUH, Majeed Ha, Shahbaz M, Kazmi F. Daily impacts of missing teeth in adult population in Lahore, Pakistan. J Pak Dent Assoc 2021;30(3):157-163.

DOI: https://doi.org/10.25301/JPDA.303.157

Received: 13 July 2020, Accepted: 26 April 2021

\section{INTRODUCTION}

$\mathrm{O}$ ral diseases being rarely life-threatening are often a low priority for health policy makers. ${ }^{1}$ Health is not merely the absence of disease but also constitutes a relationship between social and psychological wellbeing. ${ }^{2}$ World Dental Federation defines oral health as a multi-faceted ability to speak, smile, smell, taste, touch, chew, swallow and convey a range of emotions through facial expressions with confidence and without pain,

1. Assistant Professor, Department of Community and Preventive Dentistry, Rashid Latif Dental College, Lahore, Pakistan.

2. Assistant Professor, Department of Community and Preventive Dentistry, Rashid Latif Dental College, Lahore, Pakistan.

3. Demonstrator, Department of Oral Biology, Rashid Latif Dental College, Lahore, Pakistan.

4. Associate Professor, Department of Operative Dentistry, Rashid Latif Dental College, Lahore, Pakistan.

5. Assistant Professor, Department of Oral Biology, Rashid Latif Dental College, Lahore, Pakistan.

6. Professor, Department of Oral Pathology, Rashid Latif Dental College, Lahore, Pakistan.

Corresponding author: "Dr. Hazik B. Shahzad" < hazikshahzad@ hotmail.com > discomfort and disease of the craniofacial complex. ${ }^{3}$ Oral diseases including untreated caries, severe tooth loss and severe periodontitis, were listed among the top 100 Global Burden of Diseases in 2010, and severe tooth loss ranked at number $36 .{ }^{4}$ These oral diseases collectively affect 3.9 billion people worldwide. ${ }^{4}$ The World Health Organization's (WHO) Global Oral Health Programme has identified dental caries, periodontal diseases, and dental trauma as the main causes of tooth loss. ${ }^{5}$ Loss of permanent teeth is associated with significant chances of mesial drifting of neighbouring teeth to fill the empty space. ${ }^{6}$ Eventually tooth loss may results in further carious teeth and periodontal disease. Teeth are important for improving aesthetics and their loss is also responsible for functional impairments in the form of chewing limitations. ${ }^{7}$ This century has seen a shift from infectious diseases to non-communicable diseases. ${ }^{8}$ Treatments for chronic diseases are mostly management of symptoms without eradication of the disease. This elevates the need for subjective outcome measures. ${ }^{8}$ Clinical indicators alone are not adequate to describe health status. ${ }^{9}$ It has been seen 
that people with chronic diseases can observe their quality of life as being better than healthy individuals. ${ }^{10}$

Quality of Life (QoL) is defined as "an individual's perception of their position in life in the context of the culture and value systems in which they live and in relation to their goals, expectations, standards and concerns". ${ }^{11}$ With aging population, there should be adequate resources for improving QoL. ${ }^{12}$ It is a multi-dimensional and comprehensive concept that contains both positive and negative aspects of a person's physical or psychological states, independence levels, social relationships, personal beliefs and environmental features. ${ }^{11}$ In epidemiological studies, measure of QoL along with clinical measures complement perceived needs of the population. ${ }^{12}$ Subjective measures provide important information to a patients functional, social and psychological wellbeing. ${ }^{13}$ Subjective measures allow healthcare professionals to evaluate the efficacy of treatment options from the viewpoint of a patients. ${ }^{14}$ QoL is gradually acknowledged as a valid and significant indicator of service need and intervention outcomes in research and practice. ${ }^{15}$ Several instruments are currently in used to assess subjective oral health issues, hence Oral Health Related Quality of Life (OHRQoL). ${ }^{16}$ They also help us to understand the influence on oral health as well as clinical interventions on patients' wellbeing, at individual and population level. ${ }^{17}$ Factors such as gender age, or cultural background of the patient play an significant role in the perception of health. ${ }^{18}$ Other variables such as demographic, socio-economic, dental care use, may also affect the subjective perceptions of OHRQoL. ${ }^{19,20}$ Young people might consider aesthetics (staining, holes, malalignment) to be more relative to their OHRQoL. However, functional teeth for eating and speaking may be far more important for elderly. ${ }^{16} \mathrm{It}$ is thus important to obtain knowledge of what people perceive about their oral health in order to direct health strategies to provide treatment of oral diseases and rehabilitation in cases of tooth loss. ${ }^{21}$

In Pakistan, WHO in its latest 2004 report for 12-15 year old children, states the DMFT (Decayed missing and filled teeth) value to be $1.38 .^{22}$ There is little information about the oral health of adults in Pakistan. Around $90 \%$ of oral diseases in Pakistan remain untreated. ${ }^{23}$ In Pakistan, health services give least importance to oral conditions and treatment given is usually palliative or symptomatic only. ${ }^{23}$ The adult population does not seek dental treatment due to a lack of perceived requirements. ${ }^{22}$ These lacking perceptions of need, or "absence of toothache", delay required treatments until a severe painful condition arises. ${ }^{24}$ Many countries have seen a significant reduction in the prevalence of toothloss, but this oral condition still represents a significant health issue among adults in Pakistan. This study aims to find the link between impacts of toothloss and OHRQoL, that is how missing teeth impact the daily life of people. The study sample was calculated to be 372 participants. It was hypothesized that tooth loss is associated with an impairment of OHRQoL. This would enable the development of clinical decisions in public health to provide better oral health care.

\section{METHODOLOGY}

This cross-sectional research was conducted at the out-patient department of Rashid Latif Dental Hospital, Lahore. It was completed in 4 months (March 2019 to June 2019) with a sample size (n) of three hundred and seventy tree individuals aged between 18-80 years. Ethical permission was obtained from Rashid Latif Dental College Research Department. (Ref No. RLDC/001344/19). Verbal consent was taken from all participants before clinical examination and questionnaire. All participants were informed of their voluntary participation, data protection and option of opting out at any time. The size of the sample was calculated based on an expected prevalence of $30 \%$ and $95 \%$ confidence interval (CI) and $\mathrm{z}$ value of 1.96 level. ${ }^{25} \mathrm{~A}$ minimum sample size of 322 people was calculated and further increased to account for possible losses. Inclusion criteria comprised random selection of all adult patients coming to the outpatient department, having at-least ten teeth aged between 18 80 years, patients with systemic illness and people refusing to take part in the study were excluded. Three examiners were trained and calibrated against a gold standard to perform all oral examinations following WHO guidelines. ${ }^{26}$ Intra-observer agreement after 2 days of examining the same patients was found to be $99 \%$ for missing teeth (Kappa score $0.9)$.

After an introduction to the research, the respondents were asked about the effect of oral impacts on their daily life in the last six months. The Oral Impacts on Daily Performance (OIDP) questionnaire is founded on Locker's models of the World Health Organisation's (WHO) classification of disabilities impairments, and handicaps. ${ }^{27}$ It measures the impact of oral conditions on performing everyday activities in terms of severity of the self-reported impacts. ${ }^{27}$ Both English and other language (Urdu) version of OIDP were on hand for ease of management. Responses were coded from 0 (no effect) to 5 (severe effect). To determine the prevalence of each oral impact, original responses were dichotomised by a strict cut-off point $(\geq 3)$. The total OIDP score was calculated by adding the values for individual responses respectively, dividing by the maximum score (45) and multiplying by 100 . Thus, the score ranges between the values of 0 to 100 . Higher OIDP scores represent poorer OHRQoL. The total OIDP score (0-100) was divided into three categories of low impact score 
Bari YA/ Waqar SM/ Nasir S/ Zafar K/ Baig NN/ Shoro FN/ Abid K/
Daily impacts of missing teeth in adult population in Lahore, Pakistan
(0-33.3), medium impact score (33.4-66.6) and high impact score (66.7-100). Ordered Logistic regression was run as all three categories of the score were equal.

The intraoral examinations were performed on dental chair under dental unit light, using mouth mirrors and probes as recommended by the WHO. ${ }^{26}$ Each missing tooth was entered into its respective box on the scorecard. Cause of missing teeth was not considered as missing due to caries, mobility or trauma would have similar impacts. Third molars, bridges and edentulous patients were not considered. For regression analysis missing teeth were simply dichotomised into the basic two categories of either having missing teeth or not. For purposes of Chi square and trend the number of missing teeth for each person was divided into varying categories based on few, more or many missing teeth. Table 1 shows further details of the categorization. The other clinical variables used were number of carious teeth, previously filled teeth and other oral conditions including crowns, bridges, periodontal status and impactions were recorded. Demographic variables included gender and marital status. The independent variables studied were categorized.

Table 1: Categorization of Number of Missing Teeth

\begin{tabular}{|c|c|c|}
\hline Number of Missing Teeth & $\begin{array}{c}\text { Categories } \\
\text { in STATA }\end{array}$ & Categories \\
\hline 0 & 0 & None \\
\hline $1-2$ & 1 & Few \\
\hline $3-5$ & 2 & More \\
\hline $6-10$ & 3 & Many \\
\hline $11-M a x$ & 4 & Max \\
\hline
\end{tabular}

Age was divided into three groups: young adults 18-30, middle age adults 31-50, and older adults 51-80 years old. Education was classified into four groups: "Primary," "Secondary," "University" and "No education". Occupations were classified into four groups: Manager, Employed, Manual Labour and Unemployed. All data collected was entered into statistical software package STATA-14 (STATA Corp, College Station, Texas, USA) for further analysis. Chi2 for trend and Mantel-Haenszel (MH) analysis were used to determine the association of difference of impacts for participants having missing teeth compared to participants with normal number teeth, along with ordered logistic regression. 95\% significance level (p-value <0.05) was selected for P-value.

\section{RESULTS}

A final sample consisted of 373 participants. The sample had more female participants (68.7\%) as compared to male participants. The mean age was 33.7 years (95\% CI $30.7-$ 36.7 ), with young adults (18-30 years) representing $57.5 \%$ and middle age adults (31-50 years) representing $31.5 \%$ of the sample. Majority of the participants were educated to some extent, and $69.8 \%$ of the sample was either unemployed or a homemaker (Table 2).

Mean number of missing teeth was found to be 1.70 (95\% CI 0.95-2.44). Among missing teeth left mandibular second molar had highest frequency of being missing with

Table 2: Characteristics and Socio-demographics of study sample $(n=373)$

\begin{tabular}{|l|l|l|}
\hline Variable & Category & N (\%) \\
\hline Gender & Male & $117(31.3 \%)$ \\
\hline & Female & $256(68.7 \%)$ \\
\hline Age & Young Adults & $215(57.5 \%)$ \\
\hline Education & Middle Age Adults & $117(31.5 \%)$ \\
\hline & Older Adults & $41(11.0 \%)$ \\
\hline & Secondary & $102(27.4 \%)$ \\
\hline Occupation & Manager & $116(26.0 \%)$ \\
\hline & Employed & $\mathbf{5 5}(15.1 \%)$ \\
\hline & Manual Labour & $62(16.4 \%)$ \\
\hline & Unemployed & $\mathbf{2 6 0}(69.8 \%)$ \\
\hline & & $5(1.3 \%)$ \\
\hline
\end{tabular}

$17 \%$ of the total number of samples. Followed by left and right mandibular 1st molar with $14 \%$ each of the total sample.

The mean OIDP score among participants was 23.43 (95\%CI 17.1-29.7). Using the strict cut-off point of impacts scored 3 and above (OIDP $\geq 3$ ), $63.1 \%$ of the participants were showing oral impacts. The highest prevalence was found for difficulty eating $(52.1 \%)$, followed by difficulty in relaxing/sleeping $(30.1 \%)$. However, $36.9 \%$ of the population was free from severe oral impacts and only $22.1 \%$ experienced more than 5 different oral impacts scored 23 . Table 3 shows the prevalence for all the oral impacts due to teeth loss. 
Bari YA/ Waqar SM/ Nasir S/ Zafar K/ Baig NN/ Shoro FN/ Abid K/

The bivariate association was performed between each OIDP impact score and number of missing teeth. Table 4 shows the relation between OIDP impacts and number of

Table 3: Prevalence and mean OIDP reported for severity $<3(n=373)$

\begin{tabular}{|l|c|}
\hline Item & OIDP $\geq 3$ \\
\hline Difficulty eating & $52.1 \%$ \\
\hline Difficulty speaking & $12.3 \%$ \\
\hline Difficulty cleaning teeth & $21.9 \%$ \\
\hline Difficulty going out & $10.9 \%$ \\
\hline Difficulty relaxing & $30.1 \%$ \\
\hline Difficulty smiling/laughing & $24.6 \%$ \\
\hline Difficulty carrying out work & $19.1 \%$ \\
\hline Difficulty with emotional stability & $21.6 \%$ \\
\hline Difficulty enjoying contact with others & $19.1 \%$ \\
\hline Overall & $63.1 \%$ \\
\hline Mean Score (95\% CI) & $23.43(95 \% \mathrm{CI} \mathrm{17.1-29.7)}$ \\
\hline
\end{tabular}

Table 4: Chi square test for trend for Number of Missing Teeth and each Impact on daily performance, and Mantel-Haenszel Analysis with crude and adjusted ratios (* for significant values) $(n=373)$

\begin{tabular}{|l|l|l|l|}
\hline & \multicolumn{3}{|c|}{ OIDP $\geq 3$} \\
\hline & $\begin{array}{l}\mathrm{Chi}^{2} \text { for } \\
\text { Trend }\end{array}$ & $\begin{array}{l}\text { MH Odds } \\
\text { Crude }\end{array}$ & $\begin{array}{l}\text { MH Odds } \\
\text { Adjusted }\end{array}$ \\
\hline Difficulty eating & $\mathrm{P}=0.04^{*}$ & $1.04^{*}$ & $2.14^{*}$ \\
\hline Difficulty speaking & $\mathrm{P}=\mathbf{0 . 0 5 ^ { * }}$ & $1.49^{*}$ & 1.33 \\
\hline Difficulty cleaning teeth & $\mathrm{P}=\mathbf{0 . 0 6}$ & $1.41^{*}$ & 1.0 \\
\hline Difficulty going out & $\mathrm{P}=\mathbf{0 . 0 7}$ & $1.07^{*}$ & 1.05 \\
\hline Difficulty relaxing/sleeping & $\mathrm{P}=\mathbf{0 . 0 1 *}$ & $1.27^{*}$ & $1.63^{*}$ \\
\hline Difficulty smiling/laughing & $\mathrm{P}=\mathbf{0 . 0 8}$ & $1.32^{*}$ & 0.92 \\
\hline Difficulty carrying out work & $\mathrm{P}=\mathbf{0 . 0 8}$ & 0.9 & 0.7 \\
\hline Difficulty with emotional stability & $\mathrm{P}=\mathbf{0 . 0 5 *}$ & $0.78^{*}$ & 0.77 \\
\hline $\begin{array}{l}\text { Difficulty enjoying contact with } \\
\text { others }\end{array}$ & $\mathrm{P}=\mathbf{0 . 0 7}$ & 0.99 & 0.75 \\
\hline
\end{tabular}

missing teeth using the Chi-squared for trend and MantelHaenszel $(\mathrm{MH})$ analysis. Chi-square for trend showed that higher number of missing teeth significantly affected quality of life as difficulty eating, difficulty speaking and difficulty relaxing/sleeping. Furthermore, MH odds showed significant difficulty in eating and relaxing/sleeping only. For ordered regression analysis between the three ordered categories of OIDP score and missing teeth, it was observed
Daily impacts of missing teeth in adult population in Lahore, Pakistan

that people with missing teeth are 1.33 (95\%CI 0.84-5.8) times more likely to be in the higher categories of OIDP score when compared to people without missing teeth. It indicated a significant $p$-value of 0.03 . After adjusting for sociodemographic variables and other clinical conditions, the odds ratio (OR) drops to 1.28 (95\%CI 1.4-5.9) (Table 5) and p-value was significant to be 0.01 . It was noticed that age, education and marital status did not show significant results in the analysis. Overall the results revealed that tooth-loss is directly associated to higher OHRQoL score which means it caused major effects in everyday life.

Table 5: Ordered Logistic regression for association between Missing Teeth (Yes/No) and Categories of OIDP score

(Low, Medium, High): Odds ratio, 95\% confidence intervals, $\mathrm{p}$-value $(\mathrm{n}=373)$

\begin{tabular}{|c|c|c|c|}
\hline Variable & Odds Ratio & $95 \%$ CI & p-value \\
\hline Missing Teeth & 1.28 & $1.04-4.55$ & $0.01^{*}$ \\
\hline Gender & 0.36 & $0.12-0.8$ & $0.02^{*}$ \\
\hline Age & 0.99 & $0.91-1.04$ & 0.40 \\
\hline Occupation & 0.36 & $0.14-0.92$ & $0.03 *$ \\
\hline Education & 1.02 & $0.56-1.82$ & 0.83 \\
\hline Marital Status & 0.70 & $0.15-3.2$ & 0.66 \\
\hline Smoking & 1.22 & $1.06-3.20$ & $0.01 *$ \\
\hline Caries & 1.37 & $1.10-2.37$ & $0.01 *$ \\
\hline Previously Filled & 1.84 & $1.54-6.31$ & 0.98 \\
\hline
\end{tabular}

\section{DISCUSSION}

At the time of the study, $44 \%$ of the sample had one or more missing tooth. ${ }^{28}$ In this study, effect of missing tooth was compared with OHRQoL on the basis of age, gender, occupation and education. In general, the association between missing teeth and OIDP was strong.

When the effect of age was compared with OHRQoL, the results showed that this did not have a significant effect on the subjects OHRQoL. These results are similar to findings in previous studies that OHRQoL was not significantly influenced by age. ${ }^{28,29}$ In previous studies men reported greater impacts on OHRQoL than women, although statistically men and women were observed clinically to have similar tooth loss prevalence. Differences in the discernment of OHRQoL between the two genders may be 
due to individual subjective notions formed through social life and personal needs. ${ }^{30}$ However, In this study women showed greater tooth loss compared to men. In Pakistani society women may still be far less socially interactive than men. Women might also show least concerns about their oral health conditions which eventually result in tooth loss. There were clear trends for managerial occupations for having low impacts and manual occupations having more impacts. Burt et al. also determined that total tooth loss was a social issue as much as being related to disease. ${ }^{31}$ This may be because socio-economic status is related to inequalities in health, and socioeconomically deprived people have higher risks of disease and suffer more from health issues. ${ }^{32}$ The results of this study showed a significant association between tooth loss and the different socio-economic groups. More oral health impacts were noticed in unemployed (69.8\%) and manual labour job individuals $(16.4 \%)$ compared to employed patients. This may be because of fact that employed patient shows more concern about oral health and manages to bear expenses for dental check-ups.

Trend could be seen that more educated people visited the hospital for treatment. Around $89 \%$ of the sample had at least some formal education. Educated individuals usually have better knowledge of their underlying disease, persuading themselves to get treatment. ${ }^{33}$ This study reported that $31.5 \%$ of educated individuals showed oral impacts when compared with less educated individuals. Previous studies suggested that people with higher education status tend to have the lowest risk for toothloss. ${ }^{34}$ However, the results of this study showed a lack of significant association between toothloss and education. Such finding may be due to a hospital setting of the research where a higher number of educated individuals came to get treated.

A study conducted by Susin et al., the early eruption of molars makes it the most susceptible tooth of the permanent dentition. ${ }^{35}$ Similarly, current study reported that the most common missing teeth are permanent molars. Difficulty eating, and relaxing/sleeping were the top reported impacts. The significant impact of smoking on missing teeth supports the opinion that tooth loss is also related to social behaviours, especially those related to oral hygiene practices, dietary habits, smoking and regular dental check-ups. These factors also cause caries and tooth loss. ${ }^{6}$ Similar to this report present study also concluded that poor oral hygiene and smoking effect oral health which eventually results in tooth loss. It was also noticed that tooth loss has a definite impact on OHRQoL of the patients. The severity of impact on OHRQoL increased with higher number of teeth loss leading to greater oral impairment. Study participants with more than 10 teeth lost showed highest OIDP score indicating higher oral impairment. This result is similar to the study reported by Batista et al., in which the impact on OHRQoL was higher with loss of more than 13 teeth. ${ }^{36}$ Similar findings of more frequent oral impacts was reported among individuals with fewer natural teeth. ${ }^{37}$ It has been already published, that the higher number of missing teeth, the more impacts on OHRQoL. ${ }^{36}$

Dentistry faces serious challenges, addressing them would require major changes in strategy. In epidemiological studies, both normative and subjective needs should be accounted for by professionals. ${ }^{38}$ Measuring OHRQoL is necessary to account for different perceptions about individual responses to the same diagnosis. ${ }^{16}$ It would be unethical to treat a person not wanting specific treatments. Subjective measures answer the missing link of why an individual wants treatment which is directly linked to the impacts on the persons daily life.

Due to the cross-sectional nature of this study, it is not suitable to evaluate causal relationships. Other limitations are regarding timeline to exposure and its impact. Potential limitations may also include change of exposure over the assessment period. OHRQoL data being self-reported varies from individual to individual. Different combination of self-reported impacts can lead to similar scores and are difficult to interpret. ${ }^{16}$ There is a chance for having recall bias.

The study may be over reporting the results as all the sample was taken from a hospital setting. Another limitation would be the demarcation for categorisation of Missing teeth used for this study. One might argue that the position of the missing tooth may have a different impact, thus may require more elaborate categorisation.

\section{CONCLUSIONS}

At the time of the study, $44 \%$ of the population had one or more missing tooth. Difficulty eating, and relaxing/sleeping were the top reported impacts with significant results. Participants with missing teeth were more likely to report higher scores and increased number of impacts on their OHRQoL than people without missing teeth. In this sample, education and age did not appear to play a role in the association between missing teeth and OHRQoL. Within the limitations of the study, it can be concluded that tooth loss has a definite negative impact on OHRQoL. As the severity of toothloss increases, the OIDP score amplified indicating higher oral health impacts.

\section{FURTHER RESEARCH}

Further research is needed to confirm these findings, 
using longitudinal studies looking at the association between missing teeth and OHRQoL along with the number and position of the missing teeth.

\section{OTHER INFORMATION}

No funding was required or obtained for this study.

\section{CONFLICT OF INTEREST STATEMENT}

Authors declare no conflict of interest in this research

\section{REFERENCES}

1. Petersen PE. Global policy for improvement of oral health in the 21st century-implications to oral health research of World Health Assembly 2007, World Health Organization. Community dentistry and oral epidemiology. 2009;37:1-8.

https://doi.org/10.1111/j.1600-0528.2008.00448.x

2. Martins AMEdB, Jones KM, Souza JGS, Pordeus IA. Association between physical and psychosocial impacts of oral disorders and quality of life among the elderly. Ciencia \& saude coletiva. 2014;19:3461-78.

https://doi.org/10.1590/1413-81232014198.16202013

3. Glick M, Williams DM, Kleinman DV, Vujicic M, Watt RG, Weyant RJ. A new definition for oral health developed by the FDI World Dental Federation opens the door to a universal definition of oral health. Am J Orthod Dentofac Orthopedi. 2017;151:229-31. https://doi.org/10.1016/j.ajodo.2016.11.010

4. Marcenes W, Kassebaum NJ, Bernabé E, Flaxman A, Naghavi M, Lopez A, et al. Global burden of oral conditions in 1990-2010: a systematic analysis. J Dent Res. 2013;92:592-7. https://doi.org/10.1177/0022034513490168

5. Petersen PE, Yamamoto T. Improving the oral health of older people: the approach of the WHO Global Oral Health Programme. Community dentistry and oral epidemiology. 2005;33:81-92.

https://doi.org/10.1111/j.1600-0528.2004.00219.x

6. Atieh MA. Tooth loss among Saudi adolescents: social and behavioural risk factors. International dental journal. 2008;58:103-08. https://doi.org/10.1111/j.1875-595X.2008.tb00184.x

7. Larsson P. Methodological studies of orofacial aesthetics, orofacial function and oral health-related quality of life: Malmö University, Departments of Stomatognathic Physiology and Prosthetic; 2010.

8. Santosa A, Wall S, Fottrell E, Högberg U, Byass P. The development and experience of epidemiological transition theory over four decades: a systematic review. Global health action. 2014;7:23574. https://doi.org/10.3402/gha.v7.23574

9. Sprangers MA, Aaronson NK. The role of health care providers and significant others in evaluating the quality of life of patients with chronic disease: a review. J Clin Epidemiol. 1992;45:743-60. https://doi.org/10.1016/0895-4356(92)90052-O

10. Ashing-Giwa KT, Tejero JS, Kim J, Padilla GV, Hellemann G. Examining predictive models of HRQOL in a population-based, multiethnic sample of women with breast carcinoma. Quality of life Research. 2007;16:413-28.

https://doi.org/10.1007/s11136-006-9138-4

11. Organization WH. WHOQOL-BREF: introduction, administration, scoring and generic version of the assessment: field trial version, December 1996. Geneva: World Health Organization; 1996.

12. Sischo L, Broder H. Oral health-related quality of life: what, why, how, and future implications. J Dent Res. 2011;90:1264-70. https://doi.org/10.1177/0022034511399918

13. Allen F, Steele J. Oral health-related quality of life. Textbook of Geriatric Dentistry. 2015:301.

14. Wright WG, Jones JA, Spiro III A, Rich SE, Kressin NR. Use of Patient Self-Report Oral Health Outcome Measures in Assessment of Dental Treatment Outcomes. J Pub Health Dent. 2009;69:95-103. https://doi.org/10.1111/j.1752-7325.2008.00106.x

15. Sanadhya S, Aapaliya P, Jain S, Sharma N, Choudhary G, Dobaria $\mathrm{N}$. Assessment and comparison of clinical dental status and its impact on oral health-related quality of life among rural and urban adults of Udaipur, India: A cross-sectional study. J Basic Clin Pharm 2015;6: 50-8.

https://doi.org/10.4103/0976-0105.152091

16. Tsakos G, Allen PF, Steele JG, Locker D. Interpreting oral healthrelated quality of life data. Community dentistry and oral epidemiology. 2012;40:193-200.

https://doi.org/10.1111/j.1600-0528.2011.00651.x

17. Usha G, Thippeswamy H, Nagesh L. Comparative assessment of validity and reliability of the Oral Impacts on Daily Performance (OIDP) frequency scale: a cross-sectional survey among adolescents in Davanagere city, Karnataka, India. Int J Dent Hygie. 2013;11: 28-34.

https://doi.org/10.1111/j.1601-5037.2011.00540.x

18. Ekanayake L, Perera I. The association between clinical oral health status and oral impacts experienced by older individuals in Sri Lanka. J Oral Rehabilit. 2004;31:831-36.

https://doi.org/10.1111/j.1365-2842.2004.01311.x

19. Barbato PR, Muller HN, Zanchet FN, Boing AF, Peres MA. Tooth loss and associated socioeconomic, demographic, and dental-care factors in Brazilian adults: an analysis of the Braz Oral Health Survey, 2002-2003. Cadernos de saude publica. 2007;23:1803-814. https://doi.org/10.1590/S0102-311X2007000800007

20. Silva DDd, Rihs LB, Sousa MdLRd. Factors associated with maintenance of teeth in adults in the State of São Paulo, Brazil. Cadernos de saude publica. 2009.

21. Haag D, Peres K, Balasubramanian M, Brennan D. Oral conditions 
Bari YA/ Waqar SM/ Nasir S/ Zafar K/ Baig NN/ Shoro FN/ Abid K/

and health-related quality of life: a systematic review. Journal of dental research. 2017;96:864-74. https://doi.org/10.1177/0022034517709737

22. Khan AA, Ijaz S, Ayma S, Qureshi A, Padhiar I, Sofia S. Oral health in Pakistan: a situation analysis. Dev Dent. 2004;5:35-44.

23. Niaz MO, Naseem M, Siddiqui SN, Khurshid Z. An outline of the oral health challenges in "Pakistani" population and a discussion of approaches to these challenges. J Pak Dental Assoc. 2013;21:219-26.

24. Tanwir F. Absence of toothache syndrome oral health and treatment needs among urban Pakistanis: Institutionen för odontologi/Department of Odontology; 2008.

25. Naing L, Winn T, Rusli B. Practical issues in calculating the sample size for prevalence studies. Archives of orofacial Sciences. 2006;1: $9-14$.

26. Organization WH. Oral health surveys: basic methods: World Health Organization; 2013.

27. Nuttall N, Tsakos G, Lader D, Hill K. Outcome and impact-a report from the Adult Dental Health Survey 2009. NHS Information Centre. 2011.

28. Ghani F, Khan M. Missing Teeth, Edentulous Areas and SocioDemographic Status Adversely Affect the Quality of Life Missing Teeth, Edentulous Areas and Socio-Demographic Status Adversely Affect the Quality of Life. J Pak Dental Assoc.2010;19:5-14

29. Inukai M, John MT, Igarashi Y, Baba K. Association between perceived chewing ability and oral health-related quality of life in partially dentate patients. Health and quality of life outcomes. 2010;8:116.

https://doi.org/10.1186/1477-7525-8-118

30. Batista MJ, Perianes LBR, Hilgert JB, Hugo FN, Sousa MdLRd. The impacts of oral health on quality of life in working adults. Brazilian oral research. 2014;28:1-6.

https://doi.org/10.1590/1807-3107BOR-2014.vol28.0040
Daily impacts of missing teeth in adult population in Lahore, Pakistan

31. Burt B, Ismail A, Morrison E, Beltran E. Risk factors for tooth loss over a 28-year period. J Dent Re. 1990;69:1126-30. https://doi.org/10.1177/00220345900690050201

32. Krisdapong S, Prasertsom P, Rattanarangsima K, Sheiham A. Sociodemographic differences in oral health-related quality of life related to dental caries in Thai school children. Community Dent Health. 2013;30:112-18.

33. Dowd JB, Zajacova A. Does self-rated health mean the same thing across socioeconomic groups? Evidence from biomarker data. Annals Ppidemio. 2010;20:743-49.

https://doi.org/10.1016/j.annepidem.2010.06.007

34. Haugejorden O, Klock KS, Trovik TA. Incidence and predictors of self-reported tooth loss in a representative sample of Norwegian adults. Community dentistry and oral epidemiology. 2003;31:261-8. https://doi.org/10.1034/j.1600-0528.2003.00004.x

35. Susin C, Haas AN, Opermann RV, Albandar JM. Tooth loss in a young population from south Brazil. J Pub Heal Dentis. 2006;66: $110-5$.

https://doi.org/10.1111/j.1752-7325.2006.tb02565.x

36. Batista MJ, Lawrence HP, de Sousa MdLR. Impact of tooth loss related to number and position on oral health quality of life among adults. Health and quality of life outcomes. 2014;12:165. https://doi.org/10.1186/s12955-014-0165-5

37. Lahti S, Suominen-Taipale L, Hausen H. Oral health impacts among adults in Finland: competing effects of age, number of teeth, and removable dentures. Eur J Oral Sci. 2008;116:260-6. https://doi.org/10.1111/j.1600-0722.2008.00540.x

38. Nagarajappa R, Batra M, Sanadhya S, Daryani H, Ramesh G. Relationship between oral clinical conditions and daily performances among young adults in India-a cross sectional study. J Epidemiol and Global Health. 2015;5:347-57.

https://doi.org/10.1016/j.jegh.2015.03.001 